

\section{El tema laboral en las negociaciones de los TLC: lecciones de las experiencias de Colombia frente a los TLC con los Estados Unidos y la Unión Europea ${ }^{1}$}

Por Daniel Hawkins ${ }^{2}$

\section{Introducción}

L

a política estatal de promover negociaciones para tratados de libre comercio (TLC) con los socios comerciales más importantes de Colombia ha sido uno de los ejes prioritarios de los últimos cuatro gobiernos. Fue el expresidente Álvaro Uribe Vélez el primero en formalizar negociaciones con los Estados Unidos (EE. UU.) y la Unión Europea (UE), los dos mercados más grandes del planeta, para poder ampliar las preferencias comerciales para productos colombianos y generar empleos y mayor competitividad. A pesar de los profundos desacuerdos entre académicos sobre los beneficios (Bwagwhati, 2004; Boskin, 2015; OCDE, 2002) o las desventajas de firmar este tipo de acuerdos comerciales y políticos (Scherrer, 2014; Stiglitz, 2013; Wade, 2004), los recientes gobiernos de Colombia nunca quisieron debatir sobre su estrategia de integración económica y apertura comercial, a través de acuerdos bilaterales, donde las asimetrías económicas y el

Artículo recibido en julio de 2106

Artículo aprobado en noviembre de 2016.

2 Director del Área de Investigación de la Escuela Nacional Sindical. Tiene un Ph.D. en Ciencias Sociales de la Universidad de Kassel, Alemania y un posdoctorado del Centro Global de Derechos de los Trabajadores de la Universidad Estatal del Pennsylvania, Estados Unidos. 
poder de negociación eran muy evidentes. Al contrario, el expresidente Uribe, en vísperas de concluir las negociaciones con el equipo estadounidense, en 2006, dijo que ese TLC "beneficia a todas las regiones del país y a todos los sectores de la producción” (Jiménez, 2006) y en otro discurso aclaró que él firmara a ese TLC aún sí "le llovieran rayos y centellas” (Portafolio, 2006).

El actual presidente Santos, durante su primer gobierno, tomó las riendas de Uribe en la política económica y siguió la misma línea, tratando de atraer al capital extranjero al país y aprobar los grandes TLC que llevaban años en aprietos políticos por el pésimo récord que tenía Colombia en términos de la persecución y violencia en contra de sindicalistas y los defensores de derechos humanos, además de la poca efectividad institucional en lograr hacer cumplir las leyes laborales.

Al mismo tiempo que los dos últimos presidentes de Colombia veían que sus dos TLC más importantes estaban estancados y se desesperaban en buscar la fórmula para lograr que sus trámites en el Congreso estadounidense y el Parlamento Europeo, respectivamente, dieran frutos, sus dos socios, ambos con mucha experiencia en negociar, firmar y aprobar TLC con diversos países del Sur, decidieron optar por dos estrategias sutilmente distintas que podrían comprometer al Gobierno colombiano en mejorar su protección a los derechos laborales y fortalecer su capacidad para implementar su legislación laboral. Tanto las iniciativas del Gobierno estadounidense como las promovidas por diputados del Parlamento Europeo y los funcionarios de la Comisión Europea siguieron una ruta muy experimentada que combinó capítulos sobre temas laborales dentro de los TLC con unos acuerdos laborales paralelos.

En el caso estadounidense, se complementaron el capítulo laboral (17) del TLC con un Plan de Acción Laboral (PAL) firmado por los presidentes Obama y Santos en abril de 2011. La UE, por su parte, reprodujo un capítulo sobre comercio y desarrollo sostenible, previamente encontrado en el TLC que esta entidad europea firmó con Corea del Sur, lo cual 
buscó profundizar la cooperación y el diálogo en temas laborales y ambientales, y después, el Parlamento Europeo, en junio de 2012, aprobó una hoja de ruta para el Gobierno colombiano (Resolución 2628) que sugería mejoras en diversos temas relacionados con la protección y garantía de los derechos laborales y los derechos de las víctimas en Colombia.

En términos de su capacidad para destrabar ambos TLC y lograr su aprobación definitiva, los dos modelos utilizados por el Gobierno estadounidense y la UE fueron exitosos: el TLC con los EE. UU. entró en vigencia en mayo de 2012 y el TLC con la UE se implementó parcialmente en agosto de 2013. Pero ¿hasta qué punto se puede argumentar que ambos modelos, diseñados para mejorar la protección de los derechos sociales en Colombia, tuvieron los impactos prometidos? ¿Se puede comparar las formas de negociación adoptadas por los EE. UU. y la UE con respecto a temas laborales dentro de las negociaciones amplias de sus respectivos tratados de libre comercio? Al analizar ambos modelos de negociación, ¿qué lecciones podemos aprender de esas experiencias, no tanto sobre sus impactos concretos en la protección de los derechos laborales en Colombia varios años después de su acogida, sino como manera de comprender el cómo y el porqué se utilizan estos dos modelos? Y finalmente, ¿hasta qué punto podemos comprender estos dos modelos de negociación como manifestaciones de las diferencias institucionales y estructuras sociopolíticas en los EE. UU. y la UE respectivamente?

El presente artículo pretende responder a estas reflexiones y ofrecer un análisis comparativo de los estilos y modelos de negociación de temas laborales en los TLC de los EE. UU., primero, y la UE, luego, enfocándose en la experiencia concreta de sus negociaciones con Colombia. En el país, a pesar de muchas discusiones centradas en las ventajas y desventajas de los TLC, la mayoría de ellas se han enfocado en los impactos económicos, mas no en la situación de los derechos laborales y los mecanismos utilizados para protegerlos. Este artículo pretende cambiar este enfoque. 
Primero, se examinará el PAL como acuerdo laboral paralelo para Colombia, pero también como una herramienta de negociación utilizada por el Gobierno estadounidense en numerosas ocasiones en las últimas dos décadas. Luego del análisis del PAL y la forma en que se complementa y avanza en la estrategia estadounidense de combinar mecanismos legales que contemplan posibles sanciones por violaciones laborales con acuerdos bilaterales que sean monitoreados, mas no judicializados, se examinarán las diferencias en la estrategia europea de proteger derechos y estándares laborales a través de la cooperación y diálogo entre actores gubernamentales y la sociedad civil. El artículo concluye con unas reflexiones sobre las lecciones más importantes de estas dos experiencias, como paso para entender las estructuras institucionales que moldean los modelos de negociación de temas laborales que utilizan los EE. UU. y la UE y para presionar por un cambio sustantivo en estos modelos frente a su inefectividad en brindar herramientas y mecanismos adecuados para la protección y garantía de los derechos laborales en países como Colombia.

\section{El Plan de Acción Laboral, un nuevo acuerdo para proteger y garantizar los derechos laborales en el marco de los tratados de libre comercio}

El Plan de Acción Laboral (PAL), firmado el 7 de abril de 2011 entre los presidentes Obama y Santos, fue la herramienta clave para asegurar el paso del TLC entre ambos países por el Congreso estadounidense y su posterior firma y ratificación presidencial, después de casi cinco años de estancamiento ${ }^{3}$. Antes de la firma de este plan, el TLC en cuestión había estado congelado en el Congreso estadounidense debido a los altos índices de violencia antisindical y la impunidad reinante, el precario estado de los derechos laborales fundamentales y el panorama 
general de condiciones de trabajo indecentes en Colombia. Debido a estas preocupaciones inmediatas, se diseñó el Plan de Acción Laboral (PAL) y se propuso convencer al presidente de Colombia, Juan Manuel Santos, para que se comprometiera con 37 medidas concretas sobre diez temas relacionados con la protección de los derechos de los trabajadores del país.

Con la firma del PAL se dio vía libre a la aprobación del TLC y ese mismo año, en octubre, el Congreso estadounidense y el presidente Obama firmaron dicho acuerdo, aún sin tener claro si las medidas sugeridas serían adoptadas total o parcialmente y si estas tendrían efecto sobre las realidades que se querían cambiar. Si bien el PAL fue un mecanismo exitoso para desbloquear la firma del TLC, no ha sido una estrategia ni eficiente, ni única, ni novedosa, cuando se recuerdan los muchos otros acuerdos laborales paralelos o los capítulos sobre estándares laborales que forman parte de los TLC negociados entre los EE. UU y países del Sur. El PAL fue, en realidad, otro intento más para supeditar los derechos laborales con los tratados de libre comercio que se expandieron significativamente durante la década de los 90 y a principios del siglo actual.

\section{Una breve reseña de los acuerdos laborales parale- los anteriores del PAL}

El TLC entre Estados Unidos, Canadá y México, Nafta (por sus siglas en inglés) incluyó un acuerdo laboral paralelo (conocido como el Acuerdo Norte Americano sobre Cooperación Laboral, Naalc, por sus siglas en inglés). Este acuerdo ofreció cobertura para varios derechos laborales fundamentales como la libertad de asociación, la negociación colectiva, la abolición de las peores formas de trabajo infantil, la prohibición de trabajo forzoso, además de otros derechos más instrumentales, asociados con el respeto por el salario mínimo, las jornadas laborales y estándares mínimos sobre salud y seguridad en el trabajo (Burgess, 2010; Polaski, 2004). Adicionalmente, el Naalc estableció provisiones 
para los derechos de trabajadores migrantes y para las compensaciones para accidentes en el trabajo. En total, el Naalc estableció once "principios rectores", los cuales cada signatario del TLC en cuestión se comprometió a promover de acuerdo con sus leyes laborales nacionales (Doumbia-Henry y Gravel, 2006, p.191).

Se ha planteado que el Naalc, aunque bastante limitado en la manera en que protege a los derechos laborales, sí logró abrir nuevos espacios para construir coaliciones y tomar acciones concretas para el avance de los intereses de trabajadores y el movimiento sindical, sobre todo a través de los mecanismos de quejas establecidos (Compa, 2001). Pero en términos de su efectividad para mejorar las condiciones laborales y proteger a los derechos laborales fundamentales en México, la evidencia puntea hacía un fracaso rotundo ${ }^{4}$.

Desde sus inicios, las críticas sobre la forma en que se había tratado los estándares y derechos laborales en Nafta fueron diversas y amplias, pero este modelo de acuerdo laboral paralelo al TLC, con retórica sobre los derechos laborales y mecanismos de quejas por violaciones sistemáticas a estos derechos, volvió a estar presente en varios de los TLC negociados por los EE. UU. en el siglo XXI, aunque cada uno con sus acápites especiales.

$4 \quad$ Véanse algunos de los estudios sobre las múltiples formas en las cuales se nota la falencia del Naalc para brindar una protección efectiva a los derechos de libertad sindical y en erradicar las peores formas de trabajo infantil y trabajo forzoso: Bouzas, José Alfonso Ortiz (Coord.) (2009). Evaluación de la contratación colectiva en el Distrito Federal. Friedrich Ebert Foundation. Disponible en: www.democraciaylibertadsindical. org.mx/media_files/LIBRO_BOUZAS.pdf; Unna, Carlos de Buen (2011). Collective bargaining agreements for employer protection ("protection contracts") in Mexico. Friedrich Ebert Foundation. Disponible en: www.democraciaylibertadsindical.org. mx/ media_files/Paper_Charles_De_Buen.pdf; Tilly, Chris y Alvarez Galván, José Luis (2006). Lousy Jobs, Invisible Unions: The Mexican Retail Sector in the Age of Globalization. International Labor \& Working-Class History, 70, 1-25; Worker Rights Consortium (2013, junio 18). Violations of International Labor Standards at Arneses y Accesorios De Mexico, S.A. DE C.V. (GRUPO PKC). 
En julio de 2005 el Congreso estadounidense aprobó el TLC con Centro América y la República Dominicana (Cafta-DR, por sus siglas en inglés ${ }^{5}$ ). Este TLC, además de incluir cláusulas que explican la necesidad que cada país signatario respete los derechos laborales fundamentales de la OIT, con énfasis especial en la reducción de la discriminación de género en zonas francas y la disminución de las peores formas de trabajo infantil, también instó a las partes firmantes a implementar sus leyes laborales, so pena de enfrentar sanciones comerciales y evaluaciones financieras potenciales. Adicionalmente, se incluyó un mecanismo para la cooperación laboral y la construcción de capacidades (anexo 16.5 del acuerdo), el cual priorizó cinco puntos para desarrollar la capacidad institucional, de modo que permitiera regular las relaciones laborales y hacer cumplir las leyes.

Para poder implementar este mecanismo, el Gobierno estadounidense inicialmente destinó 20 millones de dólares para que los países socios pudieran modernizar y empoderar sus ministerios de trabajo de tal forma que estos podrían "hacer inspecciones y resolver conflictos laborales con un enfoque de transparencia, objetividad y con responsabilidad" (Doumbia-Henry y Gravel, 2006, p.195); un camino bastante parecido al recorrido en el PAL con Colombia. Existen pocos trabajos académicos sobre los resultados concretos de estos esfuerzos para mejorar la capacidad institucional, sobre todo con respecto a la efectividad de los sistemas de inspección de trabajo (para una excepción, véase Schrank, 2013). Y aunque se ha utilizado el mecanismo de quejas para solicitar

Disponible en: htpp://workersrights.org/Freports/WRC\%20Findings \% 20and \%20 Recommendations \% 20re\%20Arneses \% 20y \% 20Accesorios\%20 de\%20Mexico \% 20 06.18.13. pdf; Centro de Reflexión y Acción Laboral (2013, agosto). After the Reform: Fifth report about the labor conditions of Mexico's electronics industry. Disponible en: www.fomento.org.mx/novedades/Informe2013-ingles.pdf.

5 Este TLC cubrió los siguientes países: Costa Rica, El Salvador, Guatemala, Honduras, Nicaragua, la República Dominicana y los EE. UU. 
investigaciones y sanciones a países de Centroamérica que han violado los estándares laborales contenidos en el Nafta y el Cafta-DR (México, Guatemala, Honduras y República Dominicana), solo uno —el caso de Guatemala - ha logrado llegar a la etapa final de resolución y sanción potencial. Los demás vacilaron en interminables procesos de diálogo, consultas y conferencias. Y hoy en día, casi diez años desde que aquel TLC entró en vigencia, y 22 desde la ratificación del Nafta, no hay ninguna sanción interpuesta a alguno de los países implicados por violaciones en materia laboral.

\section{¿Cómo se diferencia el PAL de los acuerdos laborales anteriores en la región?}

Es justo decir que a través la implementación del PAL, al igual que para los otros acuerdos paralelos y los capítulos laborales específicos en los TLC firmados con los EE. UU., se avanzó en plantear rutas y metas más concretas para la protección de los derechos laborales y sindicales en comparación con las anteriores estipulaciones sobre temas laborales encontradas, por ejemplo, en el Sistema General de Preferencias (GSP), la iniciativa de la Cuenca Caribeña (CBI), y el Acuerdo de Preferencias Arancelarias para los Países Andinos (Atpa), posteriormente, el Atpa/DEA 6 .

La principal diferencia entre el PAL y el Naalc y los componentes sobre estándares laborales del Cafta-DR tiene que ver con su enfoque sobre la protección a sindicalistas amenazados (tema 8) y las reformas hechas al sistema de justicia para intentar acabar con la impunidad por casos de violencia en contra de sindicalistas colombianos (tema

6 Es importante anotar que el Atpa/DEA estaba más asociado al cumplimiento de los países andinos con respecto a sus políticas de antinarcóticos, siguiendo las líneas determinadas por la DEA. Véase: Patel-Campillo, Anouk (2010). Rival commodity chains: Agency and regulation in the US and Colombian cut flower agro-industries. Review of International Political Economy, 17(1), 77-102. 
9). Adicionalmente, hubo un énfasis en el PAL sobre la necesidad de acabar con prácticas de intermediación laboral ilegal (tema 3), sobre todo en las cooperativas de trabajo asociado (CTA), las cuales violaban muchos de los derechos fundamentales de los trabajadores en beneficio de las empresas.

Se repitió el enfoque sobre el fortalecimiento institucional (tema 1), principalmente a través de la creación del Ministerio de Trabajo y la expansión y profesionalización de su planta de inspectores del trabajo. Adicionalmente, varios temas intentaron diseñar medidas legislativas que protegieran mejor las libertades sindicales (temas 2, 5 y 7). Incluso, como documento de compromiso político, el PAL logró recopilar y mejorar los enfoques incluidos dentro del Naalc y el Cafta-DR. No obstante, tener un documento bien escrito y con claridad sobre cada medida no significa que su traducción en política siguiera las mismas líneas. La principal falencia del PAL, así como ha pasado con las experiencias anteriores en la región, tiene que ver con su naturaleza voluntaria. Mientras que muchas de las medidas legislativas del PAL se establecieron como obligatorias, para asegurar que se aprobara el TLC, una vez que el presidente Obama ratificó ese acuerdo, la implementación continua de las medidas del PAL carecía de esa obligación, igual que se carecían de "criterios estándares o un mecanismo de evaluación individual”, como ha mencionado la AFLCIO en una reciente publicación (AFL-CIO, 2016, p.4).

Es así que más de cinco años después de la firma del PAL estamos ante una situación en la que a pesar de las evidentes mejoras en la regulación estatal de las relaciones laborales del país (sobre todo con respecto a la creación del Ministerio de Trabajo, la ampliación de la planta de inspectores y la modernización del sistema de inspección laboral, el fortalecimiento de los sistemas de protección de sindicalistas amenazados y el gradual avance investigativo contra los miles de crímenes cometidos contra sindicalistas colombianos), muchos de los diez temas y las 37 medidas concretas del acuerdo no se han cumplido 
a cabalidad y miles de trabajadores colombianos siguen laborando sin poder ejercer sus derechos y muchas organizaciones sindicales siguen esperando que el Ministerio y la Fiscalía actúen de acuerdo con lo establecido en el PAL, para garantizar que la actividad sindical sea protegida y fortalecida, no atacada y menospreciada, como pasa con frecuencia hoy en día.

\section{El TLC entre Colombia, Perú y la Unión Europea: ¿un avance para los derechos laborales en Colombia?}

El TLC entre Colombia, Perú y la Unión Europea, firmado en Bélgica en junio de 2012, tuvo un plazo entre las negociaciones y la aprobación política bastante distinto que la experiencia del TLC entre Colombia y los EE. UU. Originalmente, se empezaron las negociaciones entre los países andinos (Colombia, Perú y Ecuador) y la Comisión de la UE7 en enero de 2009, pero poco tiempo después Ecuador suspendió su participación, así que las negociaciones entre las tres partes restantes culminaron en marzo de 2010. En otras palabras, podríamos plantear que, dado que las negociaciones para este TLC tuvieron lugar después de la terminación de las negociaciones con el equipo estadounidense, para reflexionar sobre su estrategia de negociación, se esperaría que el Gobierno colombiano hubiera aprendido de las lecciones del TLC con los EE. UU., sobre todo de los problemas asociados a la falta de una protección adecuada de los derechos laborales y sindicales en el país, que resultaron ser el talón de Aquiles en la aprobación política de dicho TLC en Estados Unidos. Obviamente, pensar así sería, primero, sobredimensionar el poder político-económico de Colombia y Perú en estas negociaciones, y segundo, equivocarse al pensar que los modelos de negociación estadounidense habrían sido iguales que

La Comisión de la Unión Europea tiene facultad de iniciar y concluir todas las negociaciones comerciales con países socios. 
los modelos de la Unión Europea, tanto en términos de forma como de sustancia.

Aunque la historia reciente de ambos bloques comerciales ilustra su creciente preferencia para impulsar la liberalización comercial a través de la negociación de acuerdos comerciales bilaterales o regionales, en vez de la táctica prevalente durante las primeras décadas después de la Segunda Guerra Mundial (SGM) de promover negociaciones multilaterales, primero a través del GATT (Acuerdo General sobre Comercio y Aranceles) y luego con la Organización Mundial de Comercio (OMC) (Aghion, Antrás y Helpman, 2007), se ha planteado que, con respecto a su política comercial y su relación con cuestiones sociales, sobre todo en relación con estándares laborales, la Unión Europea es distinta a los Estados Unidos por el hecho de que no tiene un "modelo de TLC" (acuerdos de asociación económica) que utilice al momento de negociar acuerdos con países diversos (Woolcock, 2007). Pero quizá en años más recientes la UE, como forma de armonizar sus diversas negociaciones en camino, ha diseñado un tipo de modelo social para sus nuevos TLC.

Por cierto, aunque los TLC negociados por la UE han aumentado en número durante el nuevo siglo ${ }^{8}$, fue a partir de la Estrategia Comercial de la Europa Global, en 2006, que se pudo evidenciar cómo la Comisión Europea, entidad responsable por las negociaciones comerciales ${ }^{9}$, además de diseñar y presentar cualquier normatividad de la UE, profundizó su política de acelerar las negociaciones a través de la estrategia de promover instancias de “cooperación” en temas sociales (Van den Putte et al., 2015).

8 Vean la lista de negociaciones concluidas y TLC vigentes de la UE aquí: http:// ec.europa.eu/trade/policy/countries-and-regions/agreements/\#_other-countries (recuperado el 4 de agosto de 2016).

9 Desde el año 2009, la comisión obtuvo el dominio sobre las negociaciones de los acuerdos de inversión firmados por la UE. 
A propósito, uno de los marcos que ha distinguido la aproximación de la Comisión Europea a estándares laborales y sociales en las negociaciones de los TLC de la UE al enfoque del Gobierno estadounidense ha sido, precisamente, su preferencia por adoptar una posición que resalta instancias de cooperación entre las partes firmantes en vez de estipular posibles sanciones por la violación de provisiones laborales en los TLC (Compa, 2014). Y en años recientes, este marco cooperativo se ha ampliado, como la mejor manera de alcanzar lo que se definió como una nueva competitividad (Yencken, 2016). Pero, exactamente, icómo se diferencian estos dos modelos de TLC en relación con los estándares laborales promovidos por la UE y los EE. UU.?

\section{¿En qué se basa el supuesto marco cooperativo del nuevo modelo TLC de la UE?}

Mientras que la posibilidad sancionatoria por violación de las provisiones laborales existía desde el TLC entre los EE. UU.-Canadá-México, como ya mencionamos, desde esa misma década, los 90, la UE había propuesta adoptar una política de negociar TLC y otros acuerdos comerciales donde el diálogo prevalecía sobre el castigo (Concejo de la Unión Europea, 1999). Pero sin lugar a dudas, en términos de su acercamiento a estándares y provisiones laborales, los TLC negociados por la UE en años recientes son fundamentalmente distintos que los TLC firmados hace más de una década. Por ejemplo, los TLC firmados con Chile (2002) y México (2000) no incluyeron una mención explícita de los derechos laborales. En reemplazo, el artículo 1 de ambos acuerdos planteó el vínculo entre el respeto por los principios democráticos y los derechos humanos fundamentales y "las políticas internas y externas de las partes firmantes, constituyéndose así como un elemento esencial [...]” (Compa, 2014, p.130). No obstante, esta cláusula no existe como mecanismo legalmente vinculante (Meyer, 2005). Solo después de 2006, bajo el rubro de esa nueva estrategia "competitiva” de la UE en sus políticas de negociación y apertura comercial, se vio aparecer lo que algunos han llamado los TLC de "la nueva generación” (Yencken, 2016). 
Sobre todo, a partir de la firma del TLC entre la UE y Corea (2010) se combinó la "cláusula democrática", encontrada en los TLC de Chile y México (entre otros), con extensivas referencias sobre derechos y estándares laborales (Compa, 2014, p.130). Fue en este TLC que apareció un capítulo sobre "Comercio y desarrollo sostenible” donde se encuentran los planteamientos relacionados con temas y derechos laborales y ambientales, un aparte que reaparece en el TLC firmado con Colombia y Perú. De acuerdo con algunos analistas europeos, la inclusión de este nuevo capítulo a los más recientes TLC negociados con países en vías de desarrollo, como Colombia, ha profundizado el compromiso social en las negociaciones comerciales de la UE en tres maneras (Van den Putte et al., 2013). Primero, el alcance de las normas sociales se ha profundizado y ampliado. Mientras que en los TLC firmados a principios del presente siglo se hablaban de normas sociales como áreas de cooperación, en los TLC más recientes son referidas como derechos humanos. Segundo, en los nuevos TLC se han abierto algunas formas semijurídicas para resolver disputas laborales ${ }^{10}$.

Y tercero, los nuevos TLC incluyen instancias para la participación de actores no gubernamentales sobre cuestiones relacionados con temas laborales y ambientales (Van den Putte et al., 2015, p.2).

Es cierto que la ampliación de espacios para discutir la implementación de los TLC nuevos con respecto a disputas laborales puede profundizar la cooperación, pero no alcanza ser una sustitución comparable de mecanismos concretos que obligan el cumplimiento de derechos y estándares laborales (Compa, 2014, p.131). Y es en este punto donde se diferencian los modelos de la UE y el estadounidense, y no por ser dos opuestos irreconocibles entre la "zanahoria” y el "garrote”; hay mucha

10 De acuerdo con Van den Putte et al. (2015, p.2) en la primera instancia se puede iniciar consultas gubernamentales para discutir el asunto, y si no llega a un acuerdo, se puede crear un panel de expertos encargado de buscar una resolución. 
más sutileza en cómo se relacionan las instancias de cooperación para temas laborales del modelo de la UE y los mecanismos concretos de posibles sanciones incluidos en el modelo estadounidense.

Ya hemos analizado como el Plan de Acción Laboral (PAL) firmado por los presidentes Obama y Santos, en 2011, siguió el camino iniciado hace 22 años tras la firma de Naalc (Acuerdo Norteamericano sobre Cooperación Laboral), el cual fue un acuerdo paralelo al Nafta. Todos los acuerdos laborales paralelos - firmados durante los años restantes, incluido el PAL - carecieron de mecanismos sancionatorios y fueron, más bien, compromisos entre los Gobiernos de los países firmantes para mejorar la regulación de las relaciones laborales, protegiendo derechos laborales específicos, dependiendo del contexto concreto de cada país involucrado. Pero aunque formalmente no fueron más que acuerdos voluntarios entre gobiernos, firmados para destrabar la aprobación de los TLC en el Congreso estadounidense, todos abrían la posibilidad de que los movimientos sindicales respectivos pudieran incidir, a través del monitoreo y la presión, para que cada tema mencionado y cada compromiso adquirido fueran implementados.

En el caso del PAL, el Gobierno colombiano fue presionado a publicar informes sobre el estado de su implementación cada año y el mismo representante de Asuntos Comerciales del Gobierno estadounidense (USTR) replicó con sus propios informes sobre los avances y temas por mejorar en la implementación del PAL. Hubo consultas continuas entre las centrales sindicales colombianas, el Centro de Solidaridad de la AFL-CIO, la Escuela Nacional Sindical (ENS) y funcionarios de la Embajada Estadounidense en Colombia, así como un proceso de retroalimentación con funcionarios del Departamento de Trabajo (Usdol) de los EE. UU. y miembros del Congreso estadounidense, durante los últimos cinco años, como forma de contrastar lo dicho y publicado por el Ministerio de Trabajo de Colombia con lo percibido y publicado por el sindicalismo colombiano y las ONG asociadas. 
Al mismo tiempo se pudo depositar, en mayo de 2016, una queja formal contra el Gobierno colombiano por sus incumplimientos y violaciones al capítulo laboral del TLC con los EE. UU. (capítulo 17), presentada ante el departamento de Comercio y la Oficina de Asuntos Laborales (Ilab, en inglés) de ese país, por parte de varias organizaciones sindicales y laborales de los EE. UU. y Colombia. Esta queja no significa que los EE. UU. terminarán por sancionar al Gobierno colombiano. Pero abre la posibilidad que las violaciones laborales no queden en el olvido; que se discutan, que se investiguen y que el Gobierno estadounidense mantenga una presión sobre los gobiernos de países con demandas formales. Comparando las experiencias colombianas del TLC con los EE. UU. frente al TLC con Perú y la UE, se podría apreciar lo débil que resultan ser las instancias de cooperación asociadas con este último.

\section{¿Exactamente qué mecanismos de cooperación hay en el capítulo sobre comercio sostenible y desarrollo del TLC entre Colombia, Perú y la Unión Europea?}

Siguiendo el modelo utilizado para las negociaciones con Corea del Sur, como parte del TLC con Colombia y Perú, la Unión Europea (UE) propuso la inclusión de un capítulo en ese acuerdo donde se plantearan las protecciones y garantías sobre temas laborales y ambientales y, de modo más general, para reiterar la importancia de vincular asuntos de comercio con el trabajo y el medioambiente: el título IX sobre Comercio y desarrollo sostenible. El preámbulo de este título hace referencia a varias de las declaraciones internacionales y multilaterales más importantes sobre el medioambiente, el desarrollo y el trabajo decente, como manera de subrayar el compromiso de las partes firmantes del TLC con el fin de

promover el comercio internacional de tal manera que se contribuye al objetivo del desarrollo sostenible y para trabajar por integrar y reflejar este objetivo en sus relaciones comerciales. En particular, las partes subrayan el 
beneficio de considerar asuntos laborales y medioambientales relacionados con el comercio como parte de un enfoque global sobre comercio y desarrollo sostenible ${ }^{11}$.

El artículo 267 de dicho título propone la necesidad que las tres partes: "(b) ${ }^{12}$ fortalezcan el cumplimiento de las legislaciones laborales y medioambientales de cada parte”; “(d) fortalezcan el compromiso con los principios y derechos laborales de acuerdo con las provisiones del presente título”; y “(e) promuevan la participación pública en asuntos cubiertos bajo este título”. Preocupa, sin embargo, la falta de unos mecanismos vinculantes para asegurar que estos propósitos anunciados queden inscritos en las políticas públicas de cada firmante, es decir, en ese título no se establece ningún compromiso concreto y mucho menos obligaciones para que cada país firmante realmente "mejore" la aplicación de sus leyes laborales y las leyes sobre el medioambiente. Incluso, en el artículo 268 de ese título, sobre el derecho a regular y sobre niveles de protección, se hace explícito que cada firmante tiene el "derecho soberano [...] a establecer sus políticas y prioridades nacionales sobre el desarrollo sostenible y sus niveles propios de protección ambiental y laboral, consistente con los estándares y acuerdos reconocidos internacionalmente”. Más adelante, ese mismo artículo plantea que, en vez de establecer obligaciones concretas sobre la aplicación efectiva de las leyes en armonía con los convenios internacionales respectivos, "cada parte se esforzará para que sus leyes y políticas relevantes provean y promuevan altos niveles de protección laboral y para el medioambiente" (énfasis del autor). Pero “esforzarse” por asegurar que las leyes y políticas públicas protejan los derechos de los trabajadores y los derechos de la naturaleza suena más como una manera de "intentar" en vez de "hacer" y deja cualquier evaluación sobre el desempeño de las

11 Traducido de la versión oficial en inglés por el autor.

12 Nota del autor: todas las citas directas del texto del TLC son traducciones del inglés hechas por el autor. 
partes, en relación con este tema, sobre terreno poco firme: ¿cómo se puede determinar que un Gobierno no ha intentado que sus leyes y políticas protejan los derechos laborales y al medioambiente?

Debemos afirmar que el artículo 269 de dicho TLC plantea los compromisos de cada firmante con respecto a cinco de los derechos laborales fundamentales de la OIT: la libertad de asociación sindical y la negociación colectiva, la eliminación del trabajo forzoso, la abolición efectiva de trabajo infantil y la eliminación de la discriminación en el empleo y las ocupaciones. La importancia de resaltar los dos derechos fundamentales habilitantes, como son la libertad sindical y la negociación colectiva, no es un asunto menor, pero, teniendo en cuenta la supuesta profundización del enfoque cooperativo de los nuevos TLC de la UE, que enfatiza los Derechos Humanos en vez de solo normas sociales, es extraño que no hay ninguna mención de la Convención Europea sobre Derechos Humanos ni del Acta Constitutiva de la UE, en las cuales se otorga una fuerte protección a los derechos laborales fundamentales, como la libertad sindical y la negociación colectiva, derechos que son habilitantes y por lo tanto deben ser vistos como "los derechos civiles de los trabajadores” (Kucera, 2002). Adicionalmente, es preocupante que se haya omitido alguna referencia directa a otros estándares relacionados con el trabajo decente, por ejemplo, la legislación y aplicación de salarios mínimos, la seguridad industrial y la salud ocupacional, además de las jornadas laborales ${ }^{13}$.

La inclusión del artículo 271 del título IX a través del cual, en el punto 3 , se plantea que "las partes acuerdan promocionar las mejores prácticas relacionadas con la responsabilidad social empresarial (RSE)”, es novedoso, y si se promocionara para vincular prácticas de RSE (2015, agosto). Impactos de los Acuerdos Comerciales Internacionales: adoptados por el Estado peruano en el cumplimiento y promoción de los derechos humanos en Perú. 
al terreno concreto del diálogo social dentro de cualquier empresa, donde la gerencia establezca un diálogo continuo y permanente con sus trabajadores a través de sus organizaciones sindicales representativas, podríamos avanzar en la democratización de las relaciones laborales en Colombia sin necesidad de la acción coercitiva del Estado. No obstante, el énfasis que se hace en el mismo artículo sobre la promoción del uso de "mecanismos flexibles, voluntarios y basados en incentivos” (punto 4) podría estimular más discusiones y una mayor concertación en la resolución de disputas; pero al mismo tiempo, la falta de un marco legal tiende a dilatar cualquier intento de aminorar los impactos negativos en materia de Derechos Humanos y del medioambiente ${ }^{14}$. Adicionalmente, no se plantea cómo avanzar con políticas de RSE que generen enlaces con las comunidades ubicadas en las zonas de influencia de empresas. La RSE no puede continuar siendo una política unilateral de las empresas, a través de su diseño de códigos de conducta, que busque generar consenso y una buena imagen corporativa. Durante las negociaciones para este TLC, las partes firmantes nunca hicieron un análisis de las leyes laborales respectivas para ver cuáles no cumplen con los derechos laborales fundamentales de la $\mathrm{OIT}^{15}$, y debido a esta grave ausencia, el enfoque institucional sobre mecanismos de diálogo y cooperación encontrado en el texto se queda sin enganche concreto. Además, sin tener el posible recurso de una queja oficial, como existe en la mayoría de los TLC firmados entre los Estados Unidos y países socios del Sur (incluidos Colombia y Perú), las organizaciones sindicales y a las ONG asociadas se quedan sin un mecanismo formal para sustentar sus alegatos de violaciones laborales y sindicales.

14 La RSE es, en principio, vinculante y carece de mecanismos para sancionar a las empresas por las violaciones de derechos humanos, laborales o ambientales.

15 En el caso colombiano, este punto es especialmente relevante para los pactos colectivos y el derecho a la huelga, entendiendo el segundo derecho como parte integral del derecho a la negociación colectiva. 


\section{Mecanismos de participación de la sociedad civil: espacios manejados por el antojo del Gobierno}

En contraste, el título IX del TLC entre la UE y Colombia y Perú explícitamente plantea que ningún firmante puede tener recurso a la resolución de disputas con respecto a cualquier tema que se presenta bajo este capítulo, incluyendo a los estándares laborales (artículo 285, punto 5). Por ende, las consecuencias por no cumplir con los compromisos de este título no son claras, más allá de enfrentar cierta presión pública y escrutinio. En el caso de la presión pública e instancias de seguimiento y discusión sobre la implementación del título en cuestión, aunque existen varios espacios abiertos a través de este, en el caso colombiano, a tres años de la implementación parcial de este TLC, ninguno de ellos se ha creado y desarrollado de acuerdo con el espíritu y las sugerencias del texto del acuerdo. Por ejemplo, artículo 280 del título IX (mecanismos domésticos) proyecta que cada firmante debe consultar con los comités nacionales sobre asuntos laborales, medioambientales o de desarrollo sostenible, o si no existen, los debe crear. Estos comités pueden expresar sus opiniones y hacer recomendaciones sobre los temas que les corresponden.

En la Unión Europa estos comités son llamados Domestic Advisory Groups (DAGS) y están conformados por doce miembros, repartidos de forma equitativa, por representantes de los empresarios, representantes de las organizaciones sindicales y representantes de las ONG. El DAG europeo formado para monitorear la implementación del título IX del tratado entre Colombia, Perú y la UE ha tenido tres reuniones formales ${ }^{16}$ con representantes de la UE (Parlamentarios, funcionarios de la Comisión, etc.), además de participar en la primera (y hasta la fecha, última) reunión articulada entre miembros de la sociedad civil de Colombia, en junio de 2015 en Bogotá. Tres miembros del DAG europeo (un empresario, un sindicalista y un representante de las $\mathrm{ONG}$ ) son elegidos para

16 En febrero de 2015, junio de 2015 y abril de 2016, todas en Bruselas. 
el Comité Europeo Social y Económico (Cese). El Cese proporciona la secretaria permanente de los grupos consultivos internos (GCI).

Existe un buen nivel de transparencia en la actuación de ese DAG, con breves informes sobre las discusiones de cada reunión disponibles en la página del Cese $^{17}$, pero en el caso colombiano el proceso de conformación de su GCI dista mucho de la experiencia europea. Primero que todo, el Gobierno colombiano decidió no crear ningún GCI nuevo para tratar temas laborales, ambientales y de desarrollo sostenible. Por el contrario, se decidió dar facultad, primero, a la subcomisión de asuntos internacionales de la Comisión de Concertación de Políticas Laborales y Sindicales ${ }^{18}$ (CCPLS), para temas laborales, y segundo, al Consejo Nacional Ambiental, un espacio creado en tiempos de la inauguración de la Constitución Política de 1991, pero que no ha tenido rol alguno más allá de llenar vacíos burocráticos.

Es verdad que la subcomisión de asuntos internacionales de la CCPLS es activa y se reúne cada mes, pero en vez de componerse por representantes de la sociedad civil, predomina la incidencia del mismo Gobierno. Como planteó una funcionaria del Ministerio de Trabajo en una reciente reunión con la delegación de la UE en Colombia ${ }^{19}$, en esta subcomisión tienen asiento no solo las tres centrales sindicales del país y cinco gremios empresariales, sino funcionarios del Ministerio de Comercio, Industria y Turismo, el Ministerio de Agricultura y Desarrollo Rural, Planeación Nacional y el Banco de la República. En otras palabras, no

Véase: http://www.eesc.europa.eu/?i = portal.en.international-trade-monitoringeucolombia-peru-joint-meetings (recuperado el 8 de agosto de 2016).

18 Su rol como mecanismo para las consultas laborales asociadas con el TLC con la UE se oficializó en una publicación del MinCIT en mayo de 2016: http://www. mincit.gov.co/publicaciones.php?id $=36543$

19 Esta reunión tuvo lugar en Bogotá el 11 de mayo de 2016 y la discusión centró en los avances y problemas de la implementación del título IX del TLC entre Colombia, Perú y la UE. 
hay ninguna presencia de representantes de las ONG colombianas que trabajan temas sobre el mundo laboral y la mera presencia de funcionarios del Gobierno contradice el enfoque sobre "participación de la sociedad civil” enmarcada en el artículo 281 del título IX.

Para profundizar en el aprovechamiento de los espacios novedosos de participación social en el monitoreo de la implementación del título IX de dicho TLC, primero que todo, se deben democratizar y abrir su acceso. Adicionalmente, se debe buscar más transparencia en términos del funcionamiento de dichos espacios o GCI; un informe sobre cambios en las relaciones bilaterales de comercio a partir de la implementación del TLC no es suficiente.

Seguir el mismo modelo del capítulo sobre comercio y desarrollo sostenible encontrado en el TLC firmado con Corea para el TLC negociado con Colombia y Perú es erróneo, al menos si ese capítulo es el único donde se trata asuntos laborales y ambientales. El problema de reproducir modelos homogéneos en contextos muy diferentes es algo que se puede asociar a las estrategias adoptadas tanto por la UE como por los EE. UU. Sin embargo, para el Gobierno estadounidense, su política pragmática de suscribir acuerdos laborales paralelos a estos TLC ha sido la manera en que se intente armonizar los estándares laborales y la eficacia de la regulación laboral de los países del Sur con los planteamientos encontrados en cada capítulo laboral específico. Como ya mencionamos, la naturaleza no obligatoria de estos acuerdos paralelos los deja debilitados, sobre todo después de que se aprueban los TLC relacionados, pero al menos existen para enfocar en las muchas especificidades de cada país en términos de las dificultades de proteger derechos laborales y a implementar adecuadamente las leyes laborales.

Así fue el mismo caso en el TLC entre Colombia y los EE. UU., donde se suscribió un capítulo sobre temas laborales parecido a los otros capítulos sobre este asunto firmados por el Gobierno estadounidense para otros TLC con países de Centro y Suramérica, pero luego se vieron for- 
zados a diseñar, escribir y firmar un plan de acción laboral. En el caso de la UE, en vísperas de presiones en contra de la negociación de un TLC con un país como Colombia, donde prevalece la violencia antisindical y las graves ausencias en términos de una protección garantista de los derechos laborales de los trabajadores del país, varios diputados del Parlamento Europeo acordaron formular un documento que contendría exigencias al Gobierno colombiano relacionadas con la mejoría de su regulación laboral y la protección a los derechos laborales y humanos fundamentales. Así se creó la Resolución 2628, aprobada el 13 de junio de 2012 por la Eurocámara en la ciudad francesa de Estrasburgo ${ }^{20}$.

\section{La Resolución 2628 del Parlamento Europeo: ¿otro acuerdo laboral paralelo a un TLC?}

La Resolución 2628 del Parlamento Europeo, conocida en Colombia como la hoja de ruta del TLC entre Colombia, Perú y la Unión Europea, se puede entender como un refuerzo y ampliación del Plan de Acción Laboral Obama-Santos (PAL). En total, la Resolución 2628 comprende trece temas relacionados con los derechos laborales y sindicales $-\mathrm{y}$ un tema adicional con un enfoque sobre la implementación de la Ley de Víctimas de 2011 y la protección de los defensores de derechos humanos en Colombia-, muchos de los cuales se acercan a los compromisos adquiridos por los Gobiernos de Colombia y los Estados Unidos en el PAL, aunque en varios casos, el enfoque del Parlamento Europeo (de aquí en adelante, el PE) varía; el PAL fue más enfático en términos de sus solicitudes al Gobierno de Colombia, mientras que la Resolución 2628 propone objetivos más generales y difíciles de cuantificar.

El hilo conductor de la Resolución 2628 es el deseo del PE de asegurar que el Gobierno colombiano actúe para fomentar los derechos humanos en el

20 El contenido de la Resolución 2628 se puede consultar aquí: http://www. europarl.europa.eu/sides/getDoc.do?pubRef = -//EP //TEXT + TA + P7-TA-2012$0249+0+\mathrm{DOC}+\mathrm{XML}+\mathrm{V} 0 / / \mathrm{ES}$ 
país, garantizando que cualquier obstáculo a su libre acogimiento y expresión sea castigado o eliminado y que las y los trabajadores colombianos puedan gozar de todos los derechos laborales fundamentales en su vida de trabajo y su vida cotidiana. Adicionalmente, se plantea la necesidad de establecer un marco jurídico para "los mecanismos nacionales y de diálogo con la sociedad civil [en Colombia y Perú]”, para que la participación de la sociedad civil en la implementación y monitoreo del título IX del TLC en cuestión sea lo más democrática y transparente posible.

El origen de la Resolución 2628, como exigencia al Gobierno colombiano, se funda en la efectiva eliminación del régimen de Sistema de Preferencias Comerciales, SPG + , luego de que el TLC en cuestión entrara en vigencia, ampliando el terreno de relaciones comerciales entre los dos países andinos y la UE, pero ahora sin la obligación formal de que aplicaran efectivamente 27 de los convenios sobre Derechos $\mathrm{Hu}$ manos fundamentales (incluyendo los laborales) y el medioambiente. El sistema SPG + se componía de dos regímenes especiales: uno que favorecía los países menos desarrollados y otro que buscaba estimular el desarrollo sostenible y la gobernanza (Siroën, 2013). Junto con Colombia y Perú, entre 2009 y 2011, se beneficiaban del SPG + dieciséis países. Aunque la UE nunca ha llegado a sancionar un Gobierno de un país beneficiario de las preferencias otorgadas en el SPG + y se ha cuestionado el grado de rigor con que los 27 convenios son aplicados en ciertos países, también se ha argumentado que tanto el SPG + como el sistema de SPG estadounidense proporcionan "mayores garantías que los demás tratados de libre comercio firmados en los últimos años” (Polasky y Vyborny, citados en Siroën, 2013, p.109).

La vigencia del SPG + (para Colombia y Perú) terminó con la entrada en funcionamiento, incluso parcial, del TLC entre las tres partes. En su reemplazo, para respaldar la protección a los derechos humanos, el Acuerdo comercial incluye una cláusula análoga, suscrita por Colombia en el Acuerdo de Diálogo Político y Cooperación CAN-UE en 2003 y ratificado en 2012 por el Congreso colombiano. Esta cláusula, incluida en todos los acuerdos 
comerciales firmados por la Unión Europea, subraya la necesidad de que las naciones socias respeten los Derechos Humanos fundamentales. En caso de violación de estos derechos, se adoptarán medidas "apropiadas” que se ajusten al Derecho Internacional. Nuevamente, una palabra tan ambivalente como "apropiada" deja en un marco político muy desconcertante para activistas sociales que trabajen para la protección de los Derechos Humanos y que busquen mecanismos más eficaces que el diálogo para asegurar que se respetan estos derechos y que se castiguen las personas, instituciones o empresas que son culpables de violarlos. A más de cuatro años de la aprobación de la Resolución 2628 ¿qué ha pasado con su “implementación” y cómo debemos evaluar su efectividad como mecanismo complementario al título IX del TLC con la UE?

\section{La Resolución 2628 del Parlamento Europeo: buenas intenciones que se quedaron en el aire}

Como suele suceder con los trámites y poderes distintos de la Unión Europea (UE), el amplio marco para mejorar y profundizar en la regulación de las relaciones laborales y en la protección a los Derechos Humanos y a la protección del medioambiente, contenido en la Resolución 2628, se quedó en las buenas intenciones, en términos de su impacto concreto en el ámbito del Gobierno colombiano y, por ende, no podemos comparar los alcances de esta iniciativa con los avances asociados al PAL, aun sabiendo de las grandes limitaciones y falencias de ese compromiso político entre los Gobiernos de EE. UU. y Colombia. Aunque se estipulaba que la hoja de ruta de la Resolución 2628 debía ser aprobada por el Congreso colombiano y luego implementada a cabalidad, so pena de causar la posible revocatoria del TLC por parte de la UE ${ }^{21}$, más allá de las proclamaciones formales asociadas al TLC hubo

${ }_{21}$ Ver una nota de prensa al respecto: http://www.portafolio.co/negocios/empresas/ tlc-union-europea-entra-vigor-octubre-firma-103412 (recuperado el 8 de agosto de 2016). 
pocos esfuerzos institucionales para monitorear el nivel de apropiación que hizo el Gobierno colombiano de esta resolución. La poca efectividad en lograr que, a través de este mecanismo, se pudiera presionar a Colombia para que implementara las recomendaciones encontradas en ese documento, tiene sus raíces en dos factores, uno estructural y otro coyuntural.

Lo primero y más importante para entender la inefectividad del mecanismo de control político ejercido por diputados del Parlamento Europeo tiene que ver con sus facultades formales y el poco peso político que tienen dentro de la Unión Europea. Como se mencionó previamente, la Comisión Europea (CE) es la entidad responsable de las negociaciones de los TLC y cualquier otro acuerdo comercial y de la formulación de cualquier propuesta legislativa de la UE. El Parlamento, por su parte, puede proponer modificaciones a las propuestas legislativas enviadas por la Comisión o las cláusulas de los TLC (o el texto en su conjunto), pero su aprobación final queda en manos del Consejo de la Unión, conformado por los Estados miembros. En este escenario, y debido a las estructuras partidarias del PE, donde tradicionalmente se ha ejercido un consenso de mayoría entre los dos partidos dominantes (el Partido Popular de Europa y la Alianza de Socialistas y Demócratas ${ }^{22}$ ), existe poca posibilidad de maniobra entre diputados para posicionar temas controvertidos y mucho menos para presionar por el revocamiento de un TLC, como el de Colombia, debido al no cumplimiento de un acuerdo tipo paralelo, como la Resolución $2628^{23}$. Así, a pesar de la llamada hecha por los diputados de PE de los partidos Verde y la Alianza GUE/ $\mathrm{NGL}^{24}$ en 2009 para excluir a Colombia del acuerdo en negociación debido a las altas, recurrentes y no castigadas violaciones sistemáticas

Para un análisis de las grandes debilidades estructurales del Parlamento Europeo, véase: Watkins, Susan (2014, noviembre-diciembre). Political state of the Union. New Left Review (editorial). 
de los derechos humanos, la CE hizo caso omiso (Arroyo Picard et al., 2009, p.23).

El aislamiento del PE dentro de la estructura orgánica de la UE, al mismo tiempo en que la Comisión recibe una extensión de su control a través del Tratado de Maastricht sobre el rumbo de la UE, combinados con la crisis económica y política de la UE desde que estalló la crisis económica global de 2008, han implicado que las posibilidades reales de presionar por un cumplimiento efectivo de acuerdos como la Resolución 2628 sean casi nulas. Desde 2012, las prioridades de la UE, y por extensión el PE, han cambiado significativamente: las crisis repetitivas de las economías Pigs (Portugal, Irlanda, Grecia y España) y, sobre todo, los acontecimientos asociados a los tres fracasados salvamentos de la deuda griega por el Troika, además de la continua crisis en Ucrania, y el enfoque prioritario de las negociaciones del TTIP ${ }^{25}$, y más reciente el perplejo mediático asociado al brexit en el Reino Unido, amplificada por la continuación de la crisis migratoria en la UE, asociada al creciente flujo de refugiados de los múltiples conflictos y guerras en el Medio Oriente, han puesto a Colombia y, específicamente, el grado de protección a los derechos laborales en ese país, demasiado bajo en la escala de prioridades que enfrentan todas las instituciones de la UE. Y para acabar de complicar este panorama, las más recientes elecciones para diputados del PE, ocurridas en mayo de 2014, cambiaron el enfoque del Parlamento, con la salida de varios de los más comprometidos con la Resolución 2628.

Más allá de estas muchas distracciones que cambiaron prioridades dentro de la UE y el Parlamento, el proceso de aprobación política del TLC con Colombia y Perú reflejó, en gran parte, lo que había pasado con la demorada aprobación del TLC entre Colombia y los EE. UU. en el Con- 
greso estadounidense. En el caso de este último, tan solo a seis meses después de la firma del PAL entre los presidentes Obama y Santos, se aprobó el tratado en el Congreso estadounidense, así que no se pudo avanzar lo suficiente en la implementación de las 37 medidas formuladas en ese acuerdo bilateral.

Para el caso de la Resolución 2628, tan solo seis meses después de su formulación, el Parlamento Europeo aprobó el TLC entre Colombia, Perú y la UE (el 11 de diciembre de 2012). Y mientras tanto, en el caso del PAL, hubo un proceso de lobby continuo entre sindicatos y ONG colombianos y sus contrapartes en los EE. UU., que hizo presión para que funcionarios de Usdol estuvieran en contacto casi permanente con funcionarios del Ministerio de Trabajo de Colombia, monitoreando las muchas falencias en la implementación de las medidas del PAL; para el caso de la Resolución 2628, no se establecieron mecanismos concretos de seguimiento, monitoreo y evaluación desde el parlamento y tampoco se planteó la posibilidad de consultar con organizaciones sociales en Colombia y la UE. Varias ONG de Europa ${ }^{26}$ buscaron espacios dentro del PE y la Comisión para conversar sobre las demoras y falencias del Gobierno colombiano en cumplir con las recomendaciones de la mencionada resolución, y en marzo-abril de 2015 se logró presentar las denuncias al respecto ante varios diputados del PE en Bruselas ${ }^{27}$. Lastimosamente, dicho lobby en Europa no hizo la presión suficiente para la apertura de un diálogo formal entre el Gobierno colombiano y el movimiento sindical y las ONG asociadas. Y así, no solo ha desaparecido la Resolución 2628 en las discusiones intergubernamentales (entre funcionarios de la Comisión Europea, embajadas de países europeos

26 Sobre todo, la alianza de ONG para Colombia, OIDHACO (ubicada en Bruselas) y Transnational Institute (TNI) con sede en Ámsterdam.

27 La delegación que participó en esta denuncia y reunión en el PE consistió en representantes de dos de las centrales sindicales de Colombia (la CUT y la CTC), representantes de la Escuela Nacional Sindical, Censat Agua Viva, Oidhaco y TNI. 
en Colombia, y funcionarios del Gobierno colombiano), tampoco se ha avanzado en hacer un monitoreo serio sobre el grado de implementación del título IX del TLC, con un enfoque sobre la protección de los derechos laborales fundamentales mencionados en el texto. Adicionalmente, no se ha resuelto las falencias en la creación y sostenimiento de los espacios institucionales para la participación de la sociedad civil, delineados en el mismo título.

Entonces, luego de tres años de la implementación parcial de este TLC, nos encontramos de frente no solo con graves impactos económicos asociados a la continua dependencia de Colombia de las exportaciones de petróleo y carbón, ambos productos golpeados por la caída de los precios internacionales de los commodities, sino con una situación preocupante en la cual no se ha logrado avanzar en el traslado de las recomendaciones con respecto a la protección de los Derechos Humanos, y en particular, a los derechos laborales, encontradas tanto en el título IX como en la Resolución 2628, a la esfera política colombiana.

Y por ende, el buen discurso de los derechos encontrado en ambos documentos ha quedado en el papel, sin lograr impactar sobre el terreno concreto de las relaciones laborales en Colombia. Y esta falta de acción para la protección de los derechos humanos y laborales encontrada en los textos de los TLC y en los acuerdos laborales gubernamentales paralelos a estos TLC, en la situación concreta de Colombia y otros países donde suceden graves violaciones a los derechos de las trabajadoras y los trabajadores y de activistas defensores de los derechos, es algo que no pasa cuando el asunto se trata de la protección al capital y, sobre todo, a los inversionistas extranjeros provenientes de países que tengan un TLC. Quizá, más allá de las diferencias sutiles de los dos modelos de negociación de temas laborales en los TLC adoptados, por un lado, por los Estados Unidos, y por otro, la Unión Europea, es la gran asimetría entre la protección debida a los derechos de capital y la protección debida a los Derechos Humanos lo que más refleja lo poco que importan, en lo concreto, los segundos. 


\section{Lecciones aprendidas de la débil, pero diferenciada, protección a los derechos laborales asociadas a los TLC con los Estados Unidos y con Perú y la Unión Europea}

Para entender por qué, 22 años después de la ratificación de Nafta, sigue existiendo una brecha enorme entre las protecciones y garantías ofrecidas a los inversionistas extranjeros en los TLC y las que se ofrecen a los derechos de los trabajadores, es interesante analizar las dos maneras tan distintas como se resuelven los conflictos en, primero, la Organización Mundial de Comercio (OMC), y segundo, la Organización Internacional del Trabajo (OIT). Aunque la primera entidad multilateral existe desde hace poco más de 20 años, y la segunda fue creada en las ruinas inmediatas del final de la Primera Guerra Mundial (1919), la OMC resuelve disputas comerciales entre países socios a través de medidas que hacen cumplir con las normas, o en palabras más claras, "el procedimiento de la OMC hace hincapié en el imperio de la ley"28. La OIT, por su parte, tiene un sistema de supervisión donde los pilares son el tripartidismo y la acción voluntaria de los Estados miembros (Hepple, 2005, p.50). Sencillamente, la OMC utiliza lo que se llama "hard law" (sanciones, si son necesarias) y la OIT promueve el uso de "soft law" (persuasión a través de la posible vergüenza internacional). Es por eso que, en la Conferencia de Ministros en Singapur (1996), la OMC se comprometió a defender los estándares internacionales de trabajo, pero, a la vez, definió que la OIT fuera la entidad responsable de tratar estos mismos estándares (Hepple, 2005, p.57), una fórmula más fácil para mantenerlos sin dientes.

Las diferencias entre la forma en que se llevan las disputas entre ambas entidades multilaterales suelen replicarse cuando se trata de conflictos relacionados con las inversiones y la violación de estándares o leyes

28 Véase la página oficial de la OMC: https://www.wto.org/spanish/thewto_s/ whatis_s/tif_s/disp1_s.htm (visitada el 2 de abril de 2016). 
laborales. Y es por eso que los EE. UU., a pesar de haber recibido muchas quejas por violaciones de las leyes o estándares laborales en países con quienes comparten un TLC, nunca han impuesto ninguna sanción de mercado o una multa monetaria en contra de alguno de sus socios comerciales. Y la UE, que promueve un modelo que prioriza un acercamiento a temas sociales basado en la cooperación, nunca ha llegado a sancionar a un país socio por violaciones a los Derechos Humanos o a las leyes laborales. Al mismo tiempo, existen muchas demandas en el trámite de inversionistas extranjeros en contra de países por hacer cambios legislativos o políticos, posteriores a la firma del TLC, por considerar que dichos cambios han resultado o resultarán ser dañinos económicamente para sus empresas. Y estas demandas, a diferencia de lo que sucede con las quejas (o en el caso de la UE, las denuncias) asociadas a los capítulos laborales de TLC, son resueltas en cortes supranacionales de arbitramento, donde los árbitros son economistas o abogados expertos en comercio internacional ${ }^{29}$.

El mismo EE. UU. enfrenta una demanda muy reciente de $15 \mathrm{mil} \mathrm{mi-}$ llones de dólares, interpuesta por la empresa energética canadiense TransCanada, por la decisión tomada por el presidente Obama de suspender el proyecto de oleoducto Keystone $\mathrm{XL}^{30}$. Colombia, por su parte, enfrenta demandas millonarias relacionadas con inversionistas extranjeros con sede principal en diversos países que tienen TLC vigentes con Colombia $^{31}$, y por lo menos una de estas demandantes - la minera suiza Glencore- ha estado involucrada en varios procedimientos administra-

Para una descripción y crítica a los mecanismos de funcionamiento de los tribunales de arbitraje, ver: TNI. Profiting from Injustice. https://www.tni.org/en/briefing/ profiting-injustice (recuperado el 25 de noviembre de 2016).

Véase: Democracy Now: http://www.democracynow.org/es/2016/1/7/transcanada_ sues_the_us_for_15b (visitada el 2 de abril de 2016).

31 EcoOro Minerals de Canada, usando el TLC Colombia-Canadá, por la suspensión del proyecto de minería de oro en el páramo de Santurban; Glencore de Suiza, usando el TBI Suiza-Colombia, por el cambio en la licencia de explotación 
tivos y judiciales por presuntas violaciones a las leyes y los derechos laborales fundamentales, además de haber enfrentado muchas quejas, demandas y oposición por acciones en contra de las comunidades que residen en las cercanías a sus instalaciones mineras y sus minas de carbón en el departamento del Cesar.

¿Por qué los inversionistas pueden demandar Estados por supuesto daño a su patrimonio económico mientras que trabajadores, comunidades o Estados no pueden demandar a las empresas por impactos negativos asociados a sus actividades económicas ${ }^{32}$ ? Si nunca han prosperado las demandas en contra de Estados que no protegen efectivamente los derechos laborales fundamentales, incumpliendo sus compromisos asumidos en los TLC, ipor qué miembros de la sociedad civil no pueden seguir los pasos de los inversionistas para reclamar rectificación y compensación por negligencias de su Estado o violaciones hechas por empresas que operen en su país?

Los muchos años de presión social para aumentar las protecciones y garantías para los derechos laborales de los trabajadores a través de entidades multilaterales, como la OMC, para que exijan estándares laborales mínimos en sus estatutos y asegurar que todos los TLC y otros acuerdos comerciales se rijan por estos estándares mínimos, no han logrado resultados satisfactorios. Los mecanismos han mejorado, in-

de carbón en Calenturitas; Cosigo and Tobie Mining and Energy, bajo los TLC EE.UUColombia y Canadá-Colombia, por la declaración de parque natural Yaigoji Apaporis en el Amazonas, que impide la explotación de oro; y América Movil, bajo el TLC con México, (está en proceso de notificación) por el reconocimiento de pago de infraestructura.

32 Esta pregunta se toma de un artículo escrito por un estadounidense experto en temas de estándares laborales y TLC. Véase: Compa, Lance (2016, enero 14). How to make the Trans-Pacific Partnership work for workers and communities. Disponible en http://www.thenation.com/article/how-to-make-the-transpacificpartnership-work-for-workers-and-communities/ (visitada el 30 de marzo de 2016). 
dudablemente, pero su implementación y grado de cumplimiento son parciales, en el mejor de los casos, como hemos visto en los cinco años de la implementación del PAL, y sin dientes ni legales ni políticos, en el caso del título IX del TLC con la UE y la asociada Resolución 2628 del Parlamento Europeo.

Si analizamos, de manera sintética, las sutiles diferencias, en términos de su impacto sobre la protección de los derechos laborales, del modelo estadounidense - el cual, como hemos visto, incluye un procedimiento de queja que podría resultar en una sanción a los países socios que violen las cláusulas incluidas en los capítulos laborales, además del uso de acuerdos paralelos laborales complementarios- y el modelo europeo - el cual prioriza el fortalecimiento de mecanismos institucionales de cooperación y respeto por los derechos y estándares laborales reconocidos en convenciones internacionales, sin incluir la posibilidad de sanciones comerciales o monetarias en el caso de violaciones a esas normas-, se puede decir que aunque ninguno de estos modelos ofrece una protección suficiente a los derechos laborales y ninguno pretende avanzar en integrar a políticas de trabajo decente dentro de los parámetros formales de las negociaciones para sus TLC, la experiencia colombiana demuestra que el modelo estadounidense otorga un papel más importante a las regulaciones laborales y los derechos sindicales que el modelo europeo.

La diferencia se manifiesta más clara en cómo el modelo estadounidense combina un mecanismo legal de posible sanción a un mecanismo intergubernamental de compromiso (el Plan de Acción Laboral), mientras que el modelo europeo busca el fortalecimiento de la cooperación entre actores en vez de establecer instancias concretas de castigo en el caso de violaciones claras de leyes laborales y de los derechos laborales fundamentales mencionados en los textos de sus TLC. Estas manifestaciones se visibilizan a través de las diferencias institucionales y las condiciones políticas de Estado de los EE. UU. y la entidad supranacional de la UE. 
Como se ha argumentado en otros artículos, aunque ambos modelos de negociación de acuerdos comerciales incluyen capítulos sociales donde el tema de derechos se manifiesta, las condiciones internas determinan, significativamente, la efectividad de la estrategia para proteger estos derechos (Rettberg et al., 2014). Mientras que hubo mayor apertura en el congreso estadounidense para escuchar a los sindicatos estadounidenses en contra de la firma del TLC con Colombia debido a las sistemáticas violaciones a los derechos laborales-sindicales y la violencia en contra de sindicalistas colombianos, por el hecho de que el Partido Demócrata tenía mayoría en la Cámara de Representantes entre 2006-2010, y esa presión logró congelar la aprobación del Acuerdo hasta después de que se firmara el PAL, en el caso de la UE existió poco espacio para hacer lobby político en favor de los derechos laborales en la Comisión Europea (Eberhardt, 2014), mientras que el Parlamento dio por concluido su esfuerzo por mejorar la protección de los Derechos Humanos en Colombia con la aprobación de la Resolución 2628, sin establecer mecanismos institucionales que ayudarían en lograr su efectiva implementación.

Al mismo tiempo, la naturaleza supranacional de la UE dificulta la incidencia política de movimientos sindicales y ONG, quienes deben intentar hacer lobby en diversas esferas (jurisdicciones territoriales y administrativas y organizaciones distintas). Adicionalmente, aunque el Parlamento Europeo se organiza a través de varios comités (uno de los cuales se encarga de temas laborales) a los que se encomienda elaborar enmiendas a las directivas de la Comisión, estos no tienen las facultades para hacer seguimiento a las normas aprobadas en el PE, como se da en el caso estadounidense, en el cual el Departamento de Trabajo (USDOL) y su embajada en el país respectivo se encargan de establecer mecanismos de seguimiento a la implementación tanto de los TLC como de los acuerdos laborales paralelos firmados.

Hasta el momento, con un año de diferencia en la implementación de los dos TLC en cuestión, la cooperación intergubernamental y con las sociedades civiles de la UE no ha logrado establecer una planeación, 
concertada y con medidas claras, entre los actores involucrados para avanzar en asegurar que el Gobierno colombiano (y las empresas europeas presentes en Colombia) cumplan con los dictámenes referidos a temas laborales del TLC y que hagan caso a las recomendaciones y estipulaciones encontradas en la Resolución 2628.

En el caso europeo parece que la dispersión institucional combinada con las prioridades geopolíticas de la coyuntura ha predominado mucho más que las normas sociales y las garantías discursivas sobre los derechos laborales encontrados en el TLC con Colombia y Perú. Y, obviamente, esta dispersión está reflejada en las estrategias múltiples, pero a veces sin coordinación efectiva, de los movimientos sindicales europeos, junto con diversas ONG, que se han aliado con sus organizaciones hermanas en Colombia. La experiencia de más de dos décadas del movimiento sindical estadounidense - especialmente a través de su central, la AFL-CIO y sus oficinas, ubicadas directamente en los países socios de los TLC (a través del Centro de Solidaridad) - en acompañar a los movimientos sindicales del Sur al momento de iniciar campañas e iniciativas políticas para denunciar violaciones a los derechos laboralessindicales se ha hecho notar. Su capacidad para hacer lobby en Estados Unidos, especialmente en el Congreso, y proponer y coordinar muchos de los mecanismos de seguimiento a la implementación de los TLC y, sobre todo, de los acuerdos laborales paralelos como el PAL, ha logrado un grado de efectividad que aunque dista enormemente de ser completa, es significativamente más elevada que la demostrada hasta hoy por sus contrapartes europeos. 


\section{Bibliografía}

AFL-CIO (2016). ¿Un Patrón Oro para los trabajadores? Estado de los derechos laborales en los países del Acuerdo Transpacífico de Asociación Económica. Washington D. C.: AFL-CIO.

Arroyo Picard, A.; Rodríguez, G. y Castañeda Bustamante, N. (2009, abril). The European Union: promoter of regional integration in Latin America? Rhetoric and Reality. Debate Document Alternative Regionalisms Programme. TNI: RMALC.

Aghion, P.; Antrás, P. y Helpman, E. (2007). Negotiating free trade. Journal of International Economics, (73), 1-30.

Bhagwhati, J. (2004). In Defense of Globalization. New York: Oxford University Press.

Boskin, Michael J. (2015, octubre 29). The case for the TPP. Project Syndicate. Recuperado de https://www.project-syndicate.org/commentary/tpptradegrowth-international-cooperation-by-michael-boskin-2015-10

Burgess, K. (2010). Global pressures, national policies, and labor rights in Latin America. Studies in Comparative International Development, (45), 198-224.

Compa, L. (2001). NAFTA's labour side agreement and international labour solidarity. Recuperado de Cornell University, ILR School: http://digitalcommons.ilr.cornell.edu/articles/175/

Compa, L. (2014). Labor rights and labor standards in transatlantic trade and investment negotiations: An American perspective. En C. Scherrer (Ed.). The Transatlantic Trade \& Investment Partnership (TTIP): Implications for Labor (pp. 120-136). Múnich: ICDD, Rainer Hampp Verlag.

Compa, L. (2016, enero 14). How to make the Trans-Pacific Partnership work for workers and communities. The Nation. Recuperado de http://www. thenation.com/article/how-to-make-the-trans-pacificpartnership-work-forworkers-and-communities/

Council of the European Union (1999, octubre 26). WTO: Preparation of the Third Ministerial Conference. 12121/99, 2209ª reunión del Consejo, Luxemburgo. [Comunicado de prensa]. 
Doumbia-Henry, C. y Gravel, E. (2006). Free trade agreements and labour rights: Recent developments. International Labour Review, 145(3), 185206.

Eberhardt, P. (2014). Investment protection at a crossroads. En C. Scherrer (Ed.). The Transatlantic Trade \& Investment Partnership (TTIP): Implications for Labor (pp. 100-119). Múnich: ICDD, Rainer Hampp Verlag.

Hepple, B. (2005). Labour Laws \& Global Trade. Oxford y Portland: Hart Publishing.

Jiménez, G. (2006, abril 6). El TLC traerá beneficios para todo el país: Uribe. El Colombiano.

Kucera, D. (2002). Core labour standards and foreign direct investment. International Labour Review, 141(1-2), 31-69.

Meyer, M. (2005). La implementación de la Cláusula Democrática en el Acuerdo Global México-Unión Europea un reto a futuro. En M. Atilano (Ed.) México la Unión Europea: Sociedad Civil y Gobierno. $2^{\circ}$ Foro de Diálogo Social (pp. 28-30). México: RMALC.

OCDE (2002). Foreign Direct Investment for Development: Maximising Benefits, Minimising Costs. París: OCDE.

Polaski, S. (2004, julio 14). Protecting labor rights through trade agreements: An analytical guide. Journal of International Law \& Policy, 10(1).

Patel-Campillo, A. (2010). Rival commodity chains: Agency and regulation in the US and Colombian cut flower agroindustries. Review of International Political Economy, 17(1), 77-102.

Portafolio (2006). Las ventajas y desventajas del TLC. Portafolio. Recuperado de http://www.portafolio.co/economia/finanzas/ventajasdesventajastlc-416776

Red Peruana por una Globalización con Equidad (2015, agosto). Impactos de los Acuerdos Comerciales Internacionales adoptados por el Estado peruano en el cumplimiento y promoción de los derechos humanos en Perú. Lima: RedGE. 
Rettberg, A.; De Lombaerde, P.; Lizarazo Rodríguez, L. et al. (2014, mayo-agosto). Rights, free trade and politics: The strategic use of a rights discourse in the negotiation of free trade agreements (FTAs). Colombia Internacional, 81, 129-165.

Scherrer, C. (Ed.) (2014). The Transatlantic Trade \& Investment Partnership (TTIP): Implications for Labor. Múnich: Rainer Hampp Verlag.

Schrank, A. (2013, septiembre). From Disguised Protectionism to Rewarding Regulation: The Impact of Trade-Related Labor Standards in the Dominican Republic. Regulation \& Governance, 7(3), 299-320.

Siroën, J.M. (2013). Disposiciones laborales en los tratados de libre comercio: balance y perspectivas. Revista Internacional del trabajo, 132(1), 99-122.

Stiglitz, J. (2013, julio 4). The free trade charade. Proyect Syndicate. Recuperado de https://www.project-syndicate.org/commentary/transatlantic-and-transpacific-free-trade-trouble-byjoseph-e--stiglitz

Wade, R. H. (2004). On the Causes of Increasing World Poverty and Inequality, or Why the Matthew Effect Prevails. New Political Economy, 9(2), 163-188.

Woolcock, S. (2007). European Union policy towards Free Trade Agreements. ECIPE Working Paper, n. ${ }^{\circ} 3$.

Van den Putte, L.; Bossuyt, F.; Orbie, J. et al. (2013). Social norms in EU bilateral trade agreements: a comparative overview. En T. Takacs, A. Ott y A. Dimopoulos (eds). Linking trade and noncommercial interests: the EU as a global role model. La Haya: TMC Asser Institutute

Van den Putte, L.; Orbie, J.; Bossuyt, F. et al. (2015). What social face of the new EU trade agreements? Beyond the "soft" approach. ETUI Policy Brief. European Economic, Employment \& Social Policy, (13).

Yencken, E. (2016). Lessons from CETA: Its implications for future EU Free Trade Agreements. Melbourne: University of Melbourne. Recuperado de http://www.oefse.at/fileadmin/ content/Downloads/tradeconference/ Yencken_Lessons_from_CETA_Implications_for_future_EU_FTAs.pdf 